\title{
Calibration of circular aperture area using vision probe at inmetro
}

\author{
Pedro Bastos Costa ${ }^{1, \star}$, Felipe de Oliveira Baldner ${ }^{2}$, and Fabiana Rodrigues Leta ${ }^{2}$ \\ 1 Brazilian National Metrology Institute (INMETRO), Nossa Senhora das Graças Avenue, 50 Xerém, Duque de Caxias-RJ, \\ Brazil \\ ${ }^{2}$ Fluminense Federal University, Passo da pátria street, 156 - São Domingos, Niterói-RJ, Brazil
}

Received: 8 March 2016 / Accepted: 10 April 2016

\begin{abstract}
Circular aperture areas are standards of high importance for the realization of photometric and radiometric measurements, where the accuracy of these measures is related to the accuracy of the circular aperture area calibrations. In order to attend the requirement for traceability was developed in Brazilian metrology institute, a methodology for circular aperture area measurement as requirements from the radiometric and photometric measurements. In the developed methodology apertures are measured by non-contact measurement through images of the aperture edges captured by a camera. These images are processed using computer vision techniques and then the values of the circular aperture area are determined.
\end{abstract}

Keywords: Circular aperture area, image metrology, computer vision, image processing

\section{Introduction}

The candela is the unit of measurement define as the light intensity in a given direction from a source that emits monochromatic radiation of frequency $540 \times 10^{12}$ hertz and whose intensity in that direction is $1 / 683 \mathrm{~W}$ per steradian. The International System of Units (SI), following the definition adopted by the conférence générale des poids et mesures (CGPM) since 1979, derives it from the watt [1].

Since 1979 many National Metrology Institutes have been performing the luminous intensity unit by following the detector based method, deduced from the illuminance provided by a calibrated detector and the distance between the light source and the photometer [2-5].

Among the necessary apparatus for realization of the candela it is important to point out the circular apertures which are holes with well-defined dimensions, made of anodized aluminum, with coverage of electroformed nickel, brass, or other materials. The quality of the aperture can be evaluated from the edge's sharpness and the presence of barbs. The main edges into the aperture should be machined with an angle equal to $45^{\circ}$.

Together with the distance from the light source, the area of the circular aperture is used in the measurement method. Therefore, it is important to choose a method for circular aperture measurement in which the uncertainty evaluation is well established [6]. Circular aperture measurements can be divided into two groups: contact and contactless. Measurement by contact is performed by a

^ Correspondence: pedrobcostarj@gmail.com mechanical probe system, such as coordinate measuring machines. Radiation and vision methods [7-11] are among those for contactless measurement.

This work purpose is to present the contactless measurement method for circular aperture area calibration developed at the National Institute of Metrology, Quality and Technology of Brazil, Inmetro, in order to provide traceability for radiometric measurements in Brazil, as well as for the realization of the light intensity quantity. This paper will show the proposed method based on the use of an optical probe developed by Dimensional Metrology Laboratory of Inmetro (Lamed), and will be discussed the advantages and results using this method.

\section{Measurement setup}

A displacement table, with displacements on the $x$-axis along with a column for displacement of the microscope on the $y$-axis, was used as the setup for the calibration of circular apertures. The ranges of displacement for the table are $400 \mathrm{~mm}$ for the $x$-axis and $200 \mathrm{~mm}$ for the $y$-axis. Two distinct interferometric lasers measure these displacements simultaneously. Visualization of the aperture edges can be seen through the microscope, with magnifications that can reach up to $1000 \times$. Illumination is done using a halogen lamp with controlled intensity.

The microscope had its eyepiece modified in order to have a CCD camera attached to it. The camera is connected to a computer in order to have the images captured and processed using a software developed by the 


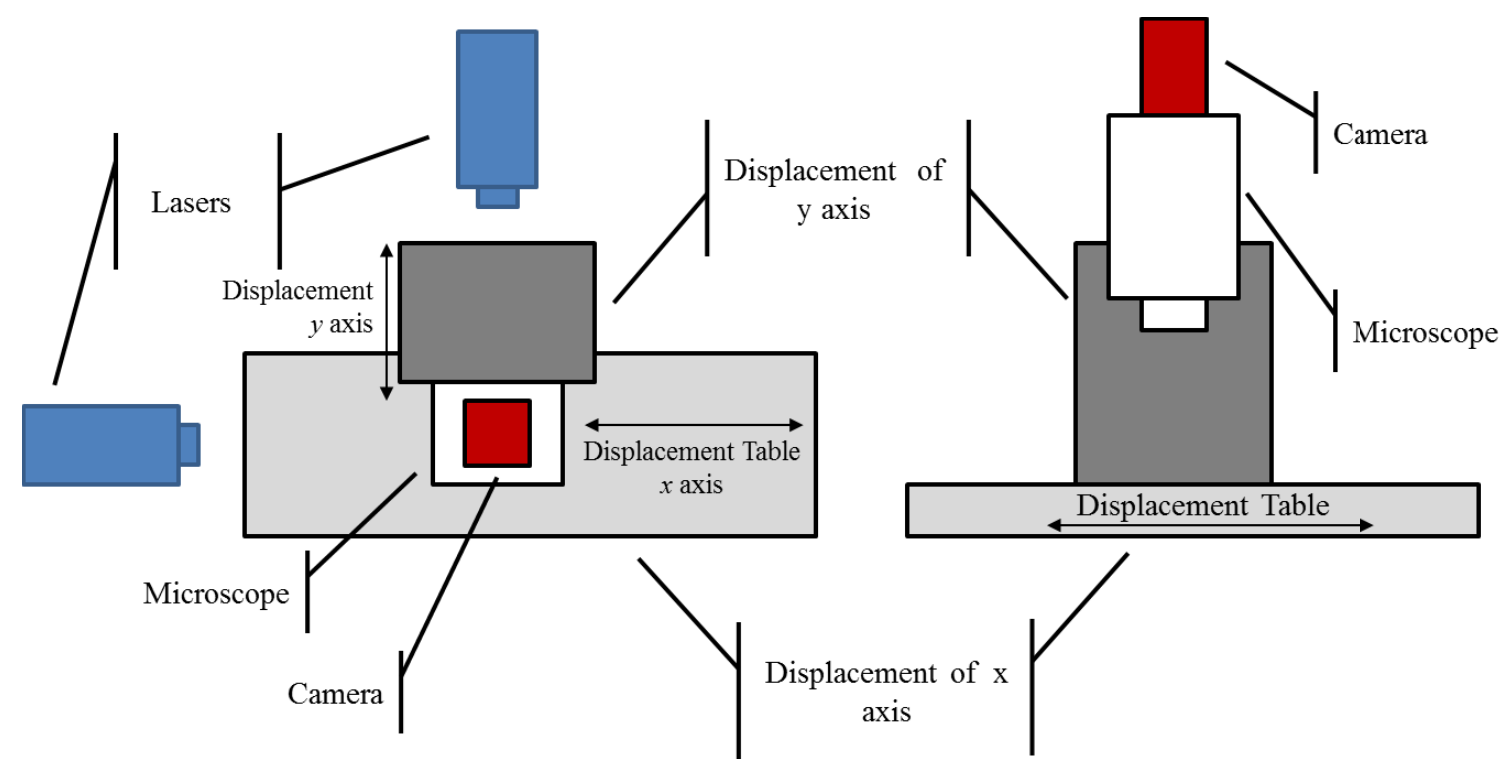

Fig. 1. Schematics of the setup with two lasers for measuring displacements and a CCD camera.
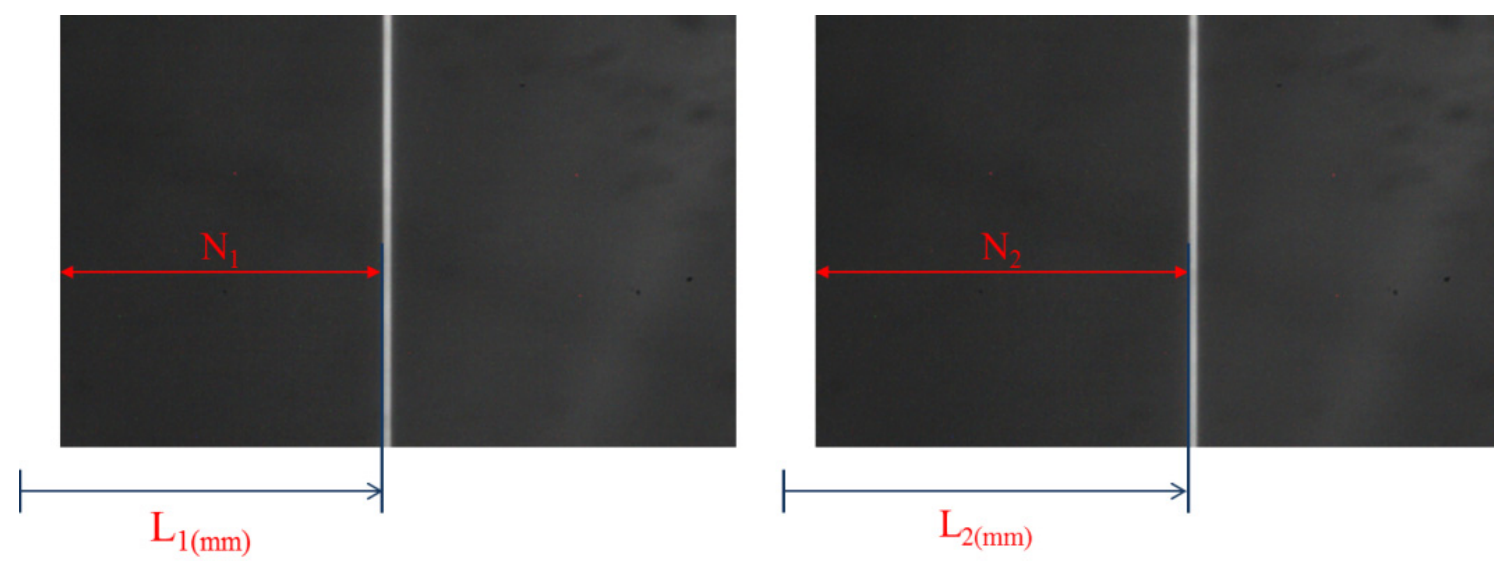

Fig. 2. Example of a sequence of images for pixels calibration.

laboratory. Filters for noise reduction and edge detection are used in this software for automatic identification of the aperture edges. A schematic of this setup is shown in Figure 1.

\section{Measuring method}

\subsection{Pixel calibration}

The first step for the measurements with the optical probe is to calibrate the pixel size in relation to the measurement conditions, that is, magnification and image resolution. This calibration will allow the conversion of a dimension measured in pixels to a value in a length unit.

A line scale is aligned in one of the measuring axis and then focused on by the microscope in the same magnification that will be later used to the calibration of the circular aperture.
The calibration then proceeds by capturing several images of the same mark in the scale, but in different positions, along with a measurement of displacement provided by the interferometric lasers, as shown in Figure 2.

The pixel length is then determined as the ratio between the displacements measured by the laser and the distance of the line scale mark for all images, as shown in equation (1). The pixel size is then determined in both $x$ and $y$ axes.

$$
l_{p(k)}=\frac{L_{2}-L_{1}}{N_{2}-N_{1}}
$$

\subsection{Image aquisition}

The aperture area measurement consists in capturing 12 images around the aperture, with intervals of $30^{\circ}$ between each image, as shown in Figure 3. 


$$
K=\left[\begin{array}{lll}
\sum_{i=1}^{n}\left[C_{X}(i)^{2}+C_{Y}(i)^{2}\right]^{2} & \sum_{i=1}^{n}\left[C_{X}(i)^{2}+C_{Y}(i)^{2}\right] \cdot C_{X}(i) & \sum_{i=1}^{n}\left[C_{X}(i)^{2}+C_{Y}(i)^{2}\right] \cdot C_{Y}(i) \\
\sum_{i=1}^{n}\left[C_{X}(i)^{2}+C_{Y}(i)^{2}\right] \cdot C_{X}(i) & \sum_{i=1}^{n} C_{X}(i)^{2} & \sum_{i=1}^{n}\left[C_{X}(i) \cdot C_{Y}(i)^{2}\right] \\
\sum_{i=1}^{n}\left[C_{X}(i)^{2}+C_{Y}(i)^{2}\right] \cdot C_{Y}(i) & \sum_{i=1}^{n}\left[C_{X}(i) \cdot C_{Y}(i)^{2}\right] & \sum_{i=1}^{n} C_{X}(i)^{2}
\end{array}\right]
$$

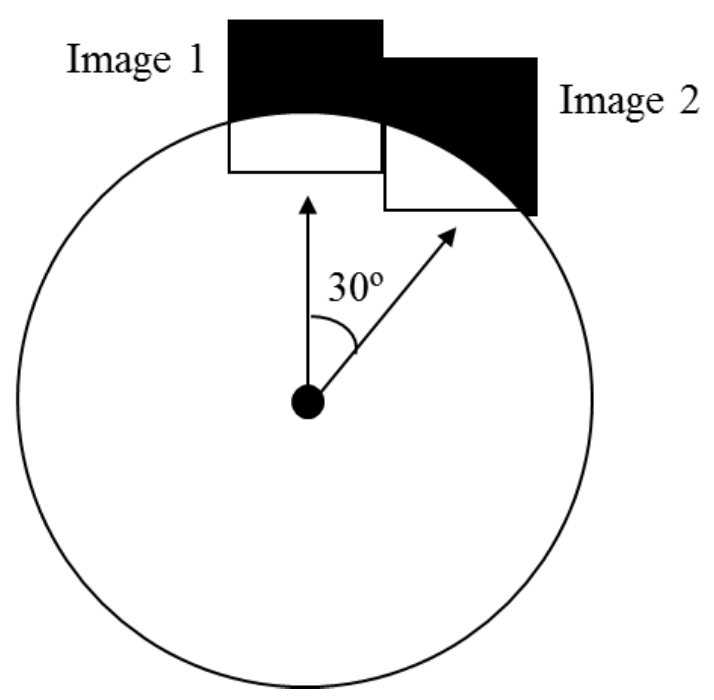

Fig. 3. Example of image acquisition around the aperture with $30^{\circ}$ interval.

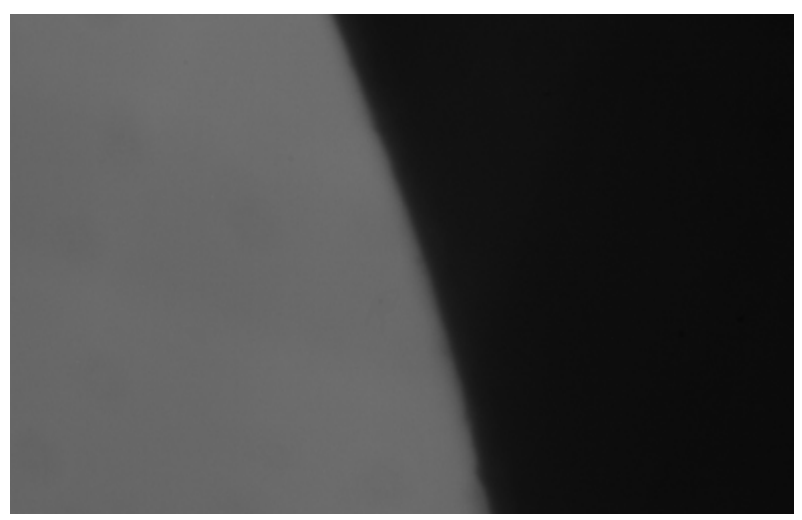

Fig. 4. Real image from the circular aperture.

For each of those 12 images, there is also a corresponding displacement value of the $x$ and $y$ axes, which is recorded as well.

\subsection{Image processing}

The aim of image processing in the circular aperture measurement is to automatically detect, all edge points in the aperture. For this purpose three techniques were used to evaluate the influence of image processing in the measurements results.

Figure 4 shows a real image captured from de circular aperture without processing.
Histogram-based segmentation was the first technique tested. From the evaluation of the image's histogram (Fig. 5a), an intensity value of 50 was chosen as the threshold between light and dark regions. The result of histogram-based segmentation is shown in Figure 5b.

The second technique tested was Otsu's method [12], obtaining the result shown in Figure 6 .

The last technique tested was Canny's method for edge enhancement [13]. Figure 7 shows the result for this method.

In order to compare the methods the variation of edge points found was assessed. In this evaluation, it was observed that regardless of the technique used the positions of the edges have not changed, so the choice of technique did not influence the measurement result.

Among the three methods, Canny's was selected for use in the measurement procedure.

\subsection{Radius results}

After the image processing step, along with the displacement measurements from the lasers, in each of the 12 images an edge point $i$ is taken. The coordinate of this edge point, in a direction $k$ ( $k$ being either $x$ or $y$ ), is a result of the product between the pixel value $\left(P_{k}(i)\right)$ and the pixel length $\left(l_{p(k)}(i)\right)$, which is then summed with the laser measurement for the $k$ axis $\left(L_{k}(i)\right)$ as shown in Figure 8 and equation (2). With this, every edge point in all 12 images has a result in length units.

$$
C_{k}(i)=L_{k}(i)+\left(P_{k}(i) \cdot l_{p(k)}(i)\right)
$$

For the determination of the aperture radius, all coordinate measurements, in $\mathrm{mm}$, around the aperture $C_{X}(i)$ and $C_{Y}(i)$ are used. These coordinates are grouped in two matrices $K$ and $J$ respectively shown by equations (3) and (4).

$$
\text { See equation (3) above. }
$$

$$
J=\left[\begin{array}{l}
\sum_{i=1}^{n}\left[C_{X}(i)^{2}+C_{Y}(i)^{2}\right] \\
\sum_{i=1}^{n} C_{X}(i) \\
\sum_{i=1}^{n} C_{Y}(i)
\end{array}\right]
$$

The coordinates corresponding to the center of the aperture in the $x$ and $y$ directions, as well as the aperture 


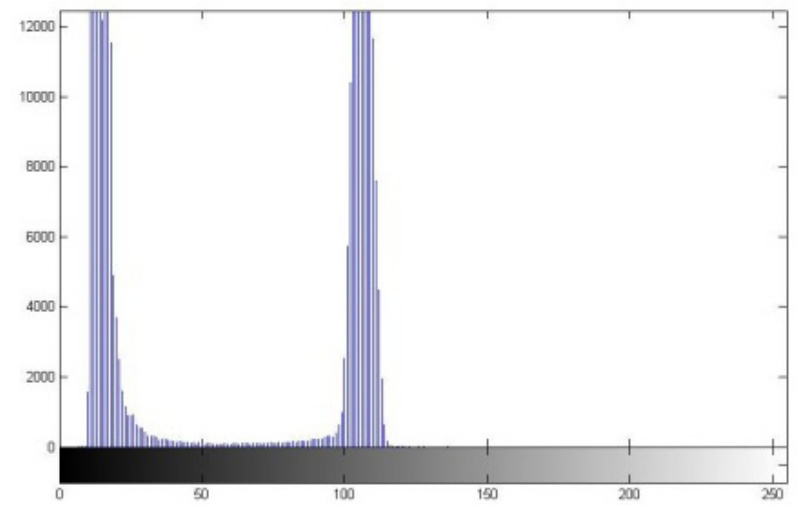

(a)

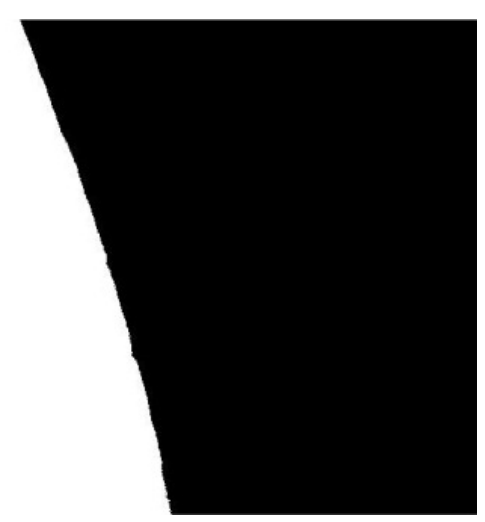

(b)

Fig. 5. Application of histogram-based segmentation.

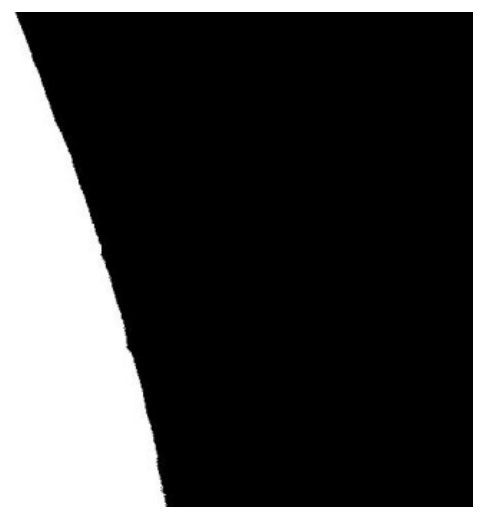

Fig. 6. Application of Otsu's method for image segmentation.

radius are obtained by solving the system of equations shown in equation (5):

$$
G=K^{-1} J
$$

where the center of the aperture, $O_{X}$ and $O_{Y}$, and the radius $R$ will be obtained using, respectively, equations (6)-(8).

$$
\begin{aligned}
O_{X} & =-\frac{G_{21}}{2 G_{11}} \\
O_{Y} & =-\frac{G_{31}}{2 G_{11}} \\
R & =\frac{\sqrt{4 G_{11}+G_{21}^{2}+G_{31}^{2}}}{2 G_{11}} .
\end{aligned}
$$

\section{Measurement results}

\subsection{Pixel calibration}

As mentioned before, the pixel calibration is performed after image acquisition and processing, allowing a conversion between pixels and millimeters for the aperture measurement. The calibration is dependent on the configuration

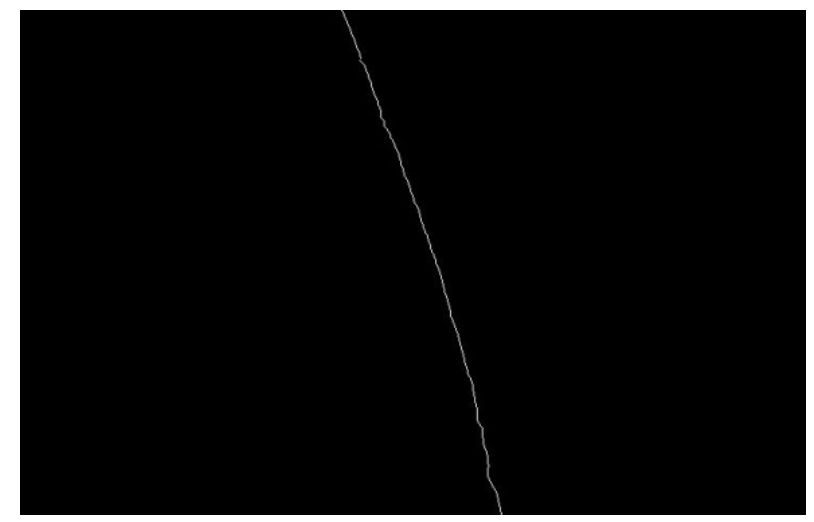

Fig. 7. Result of Canny method.

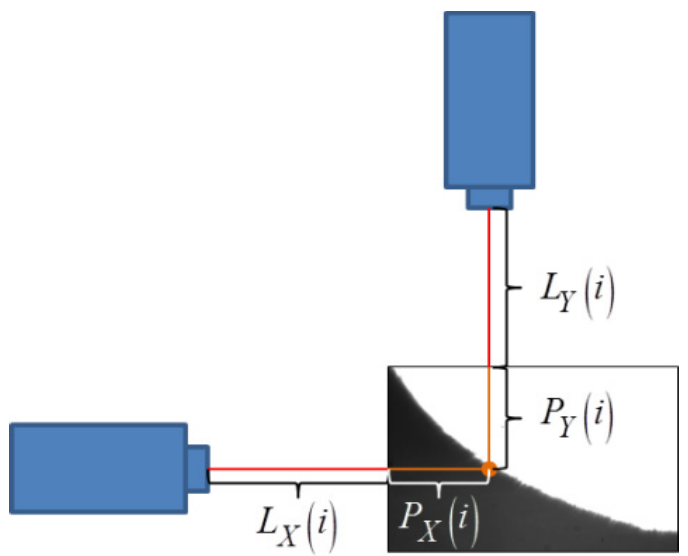

Fig. 8. Scheme of calculating pixels coordinates in length unit.

used for the aperture measurement, such as image resolution, microscope magnification and lens focus. Values for the calibration are shown in Table 1 , for magnification of $100 \times$ and resolution of $752 \times 480$ for the $x$ and $y$ axes. 

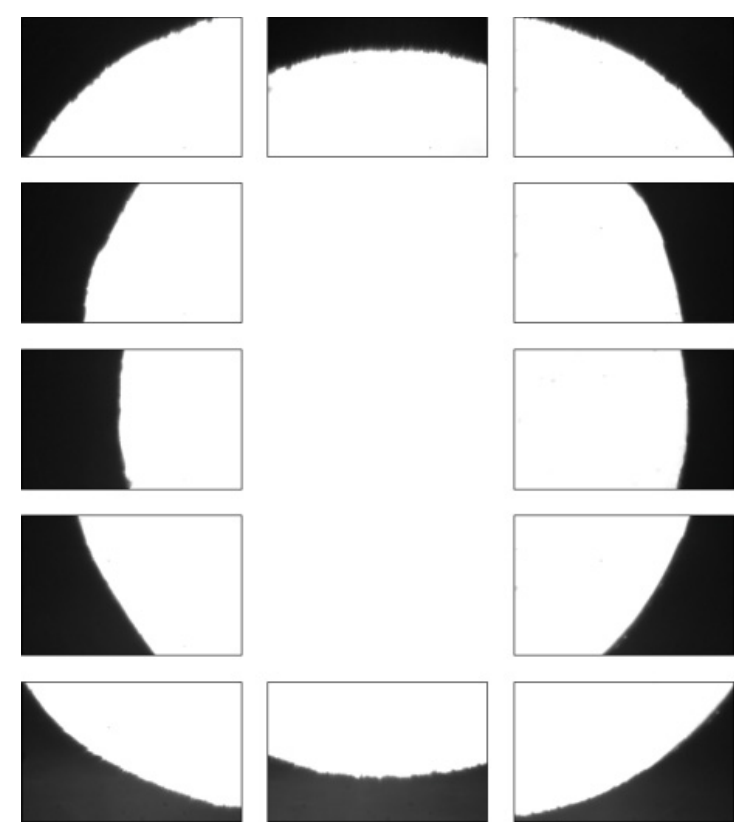

Fig. 9. Captured images of an aperture with $3 \mathrm{~mm}$ diameter.

Table 1. Results for pixel calibration with magnification of $100 \times$.

\begin{tabular}{ccc}
\hline Axes & Length $(\mathrm{mm})$ & Uncertainty $(\mathrm{nm})$ \\
\hline$X$ & 0.001334 & 20 \\
$Y$ & 0.001338 & 20 \\
\hline
\end{tabular}

\subsection{Circular aperture measurement}

The proposed methodology was applied to a circular aperture of nominal diameter of $3 \mathrm{~mm}$. The 12 images captured with increments of $30^{\circ}$ are shown in Figure 9.

\subsection{Image processing}

As described in the image processing section, Canny's algorithm was used to segment each of the captured images. The resulting images are shown in Figure 10.

\subsection{Results}

The main advantage of using the proposed method of the optical probe is the number of measurement points obtained for the measurement. In the case of the circular aperture, the 12 images generated a total of 7657 coordinate points around the circumference, obtaining a better description of any possible roundness errors.

In order to determine the repeatability of the system this procedure was repeated 5 times, thus having 60 captured images in total. For each cycle of 12 images, a radius was determined, therefore obtaining 5 measurement results the aperture areal all shown in Table 2, along with an average radius and the repeatability.

Therefore, the $3 \mathrm{~mm}$ aperture area had a results of $7.0821 \mathrm{~mm}^{2}$.
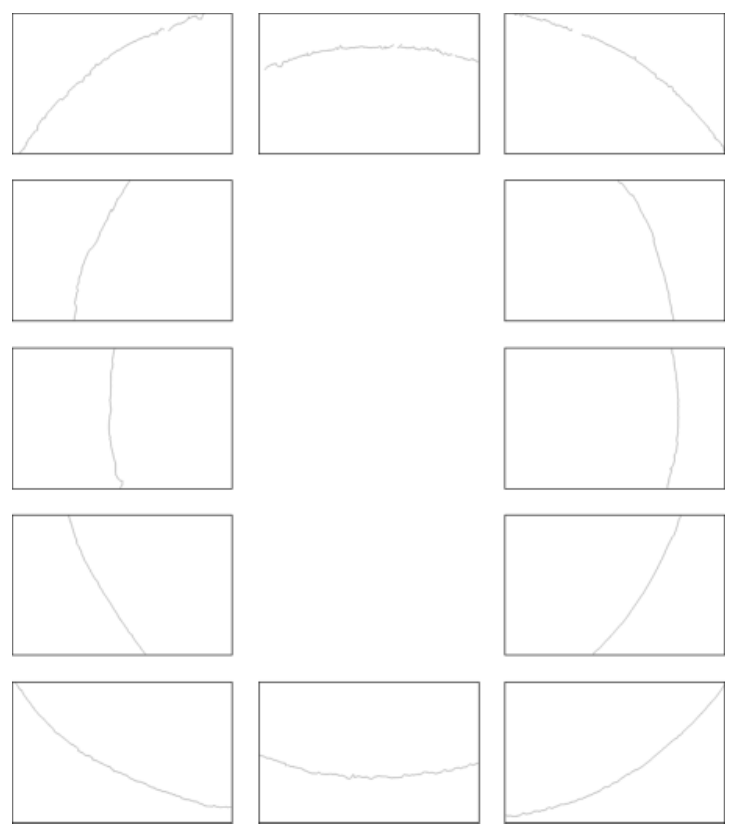

Fig. 10. Results for Canny's algorithm in the acquired images.

Table 2. Measurement results for 5 cycles.

\begin{tabular}{ccc}
\hline Cycle & Radius $(\mathrm{mm})$ & Area $\left(\mathrm{mm}^{2}\right)$ \\
\hline 1 & 1.5010 & 7.0779 \\
2 & 1.5013 & 7.0810 \\
3 & 1.5017 & 7.0847 \\
4 & 1.5015 & 7.0828 \\
5 & 1.5016 & 7.0841 \\
Average & 1.5014 & 7.0821 \\
Repeatibility & 0.0001 & 0.0012 \\
\hline
\end{tabular}

\subsection{Uncertainty of Measurement}

In order to evaluate the uncertainty of measurement was carried out a simulation using the Monte Carlo method. Each input quantity in the mathematical model (Eq. (2)) is estimated as a random variable with maximum and minimum limits defined, for uniform density function or calculating average and standard deviation for normal distributions. These parameters are shown in Table 3 .

During the simulation were realized 200.000 interactions to estimate the standard deviation for the model. The probability density function resultant from the simulation is showed in Figure 11.

In the case the standard deviation obtained was $0.0032 \mathrm{~mm}^{2}$. Expanding the result uo to $95.45 \%$ of confidence level the expanded uncertainty was $0.0064 \mathrm{~mm}^{2}$.

\section{Conclusions}

Analyzing the results enables the observation of the performance of the optic probe method developed for circular aperture measurement. Canny's algorithm for segmentation was successful in automatically determining the edge points around the opening. 
Table 3. Parameters for random variables.

\begin{tabular}{ccc}
\hline Variable & Density function & Parameters \\
\hline Laser length in $x$ axis $-L_{x}(i)$ & Uniform & $\begin{array}{c}\text { Min. limit }=-0.0001 \mathrm{~mm} \\
\text { Max. limit }=+0.0001 \mathrm{~mm}\end{array}$ \\
\hline Laser length in $y$ axis $-L_{y}(i)$ & Uniform & $\begin{array}{c}\text { Min. limit }=-0.0001 \mathrm{~mm} \\
\text { Max. limit }=+0.0001 \mathrm{~mm}\end{array}$ \\
\hline Pixel coordinate in $x$ axis $-P_{x}(i)$ & Uniform & $\begin{array}{c}\text { Min. limit }=-2 \text { pixels } \\
\text { Max. limit }=+2 \text { pixels }\end{array}$ \\
\hline Pixel coordinate in $y$ axis $-P_{y}(i)$ & Uniform & $\begin{array}{c}\text { Min. limit }=-2 \text { pixels } \\
\text { Max. limit }=+2 \text { pixels }\end{array}$ \\
\hline Pixel length in $x$ axis $-l p_{x}$ & Normal & $\begin{array}{c}\text { Average }=546 \mathrm{~nm} \\
\text { Standard deviation }=15 \mathrm{~nm}\end{array}$ \\
\hline Pixel length in $y$ axis $-l p_{y}$ & Normal & $\begin{array}{c}\text { Average }=546 \mathrm{~nm} \\
\text { Standard deviation }=15 \mathrm{~nm}\end{array}$ \\
\hline
\end{tabular}

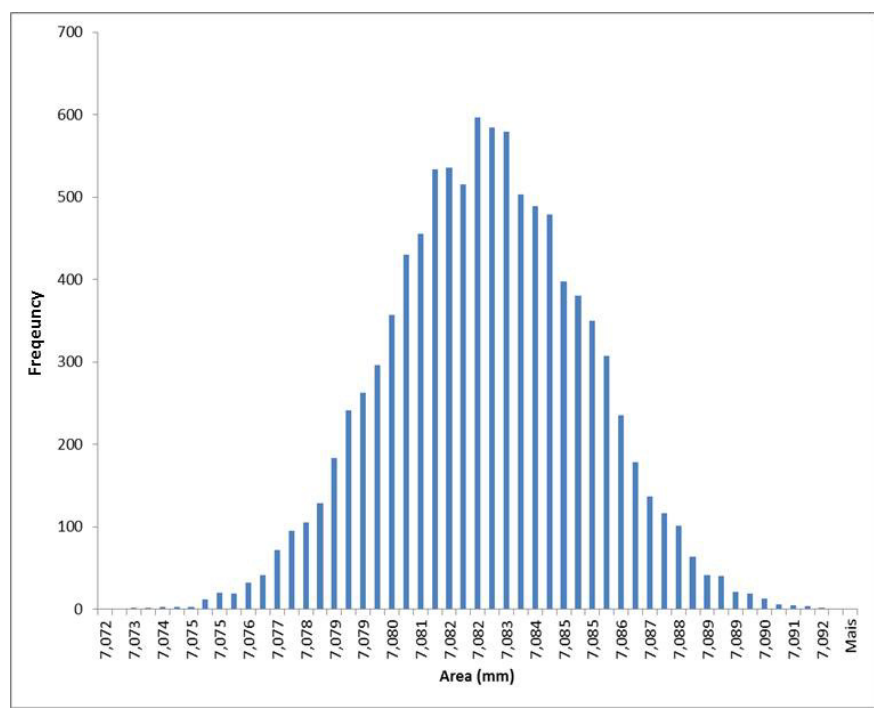

Fig. 11. Histogram resultant from the simulation.

The use of two lasers for measuring displacements in $x$ and $y$ axis simultaneously increases the accuracy of the method, since there is no need to use the machine own scales.

Another point to note to highlight is the use of $12 \mathrm{im}-$ ages around the aperture. With this, a good description of the entire aperture edge is obtained, taking into consideration any roundness deviations or imperfections that would not appear in a point-to-point measurement.

The measurement uncertainty obtained was less than $0.08 \%$ of the aperture area, being more than sufficient for the traceability of radiometric and fotometric measurements and, thus, for performing the unit of candela.

Acknowledgements. The authors would like to thank the CNPQ for the support on the research development (Reference No. 312443/2015-3).

\section{References}

1. P. Giacomo, News from the BIPM, Metrologia 16, 55-61 (1980)

2. N.P. Fox, Trap detectors and their properties, Metrologia 28, 197-202 (1991)

3. T.R. Gentile, J.M. Houston, C.L. Cromer, Realization of a scale of absolute spectral response using the National Institute of standards and technology high-accuracy cryogenic radiometer, Appl. Opt. 35, 4392-4403 (1996)

4. Y. Ohno, A handbook of applied photometry, Chapter 3: Photometric Standards (1996)

5. C.L. Cromer, G. Eppeldauer, J.E. Hardis, T.C. Larason, Y. Ohno, A.C. Parr, The NIST detector-based luminous intensity scale, J. Res. Natl. Inst. Stand. Technol. 101, 109-132 (1996)

6. L. Alves, C.T. Coelho, F. Reis, P.B. Costa, Análise da Medição da Abertura para a Realização da Candela, 1st Congresso Internacional de Metrologia Mecânica, Rio de Janeiro, Brasil, 2008

7. J.B. Fowler, High Accuracy Measurement of aperture area relative to a standard known aperture, J. Res. Natl. Inst. Stand. Technol. 100 (1995)

8. E. Ikonen, P. Toivanen, A. Lassila, A new optical method for high-accuracy determination of aperture area, Metrologia 35, 369-372 (1998)

9. G. Obein, L. Gonzalez-Galvan, J. Bastie, A New Realization of the Candela at the LNE-INM/CNAM, CIE 26TH Session, D2, Beijing, 2007, pp. 192-195

10. A. Razet, J. Bastie, Uncertainty evaluation in non-contact aperture area measurements, Metrologia 43, 361-366 (2006)

11. P.B. Costa, A. Marques, F. De Oliveira Baldner, F. Rodrigues Leta, Line scale measurement using image registration. Int. J. Metrol. Qual. Eng. 4, 121-112

12. N. Otsu, A Threshold Selection Method from gray-level histograms. IEEE Trans. Syst. Man Cybern. 9, 62-66 (1979)

13. J. Canny, A computational approach to edge detection, IEEE Trans. Patern Anal. Mach. Intell. 6, 679-698 (1986) 